\title{
Civilisations
}

Revue internationale d'anthropologie et de sciences

humaines

$48 \mid 2001$

La question de l'islam et de l'Etat à l'aube du XXIe siècle

\section{La question de l'islam en Belgique}

\section{Yassine Didier Beyens}

\section{(2) OpenEdition}

Journals

Édition électronique

URL : http://journals.openedition.org/civilisations/3474

DOI : 10.4000/civilisations.3474

ISSN : 2032-0442

\section{Éditeur}

Institut de sociologie de l'Université Libre de Bruxelles

\section{Édition imprimée}

Date de publication : 31 août 2001

Pagination : 153-159

ISSN : 0009-8140

\section{Référence électronique}

Yassine Didier Beyens, «La question de l'islam en Belgique », Civilisations [En ligne], 48 | 2001, mis en ligne le 01 août 2001, consulté le 01 mai 2019. URL : http://journals.openedition.org/civilisations/3474 ; DOI : 10.4000/civilisations.3474 


\title{
LA QUESTION DE L'ISLAM EN BELGIQUE
}

\author{
Yassine Didier BEYENS
}

\section{INTRODUCTION : UNE AUTRE APPROCHE DU MULTICULTURALSME}

Le débat autour de la question multiculturelle peut s'orienter à travers une vision sociologique (quels sont les facteurs sociaux et politiques qui règlent les relations et les enjeux de pouvoir entre les différences ?) ou juridico-politique (quelles sont les solutions juridiques et politiques relatives aux droits et devoirs reconnus aux minorités ?). Comme il peut s'inscrire dans une orientation plus philosophique et abstraite (l'unité peut-elle s'accommoder de l'hétérogénéité ?), ou, au contraire, pragmatique et concrète (comment gérer et atténuer les différends engendrés par la mixité des différences dans un contexte donné ?).

Dans ce dernier cas, il faut partir de situations réelles, admettre aussi bien la coexistence de groupes ethniques, culturels et religieux différents dans une même société, que l'émergence conséquente de différends, pour finalement essayer de promouvoir des pistes de communication et d'échange.

La démarche est réaliste car elle ne nie pas les différences. En tout cas, elle ne s'en offusque pas. Elle est aussi humaniste et idéaliste car elle suppose une nécessité de gérer les conflits et une possibilité de les atténuer autrement qu'en favorisant une catégorisation binaire qui davantage creuse le fossé qu'il n'éveille à le combler.

Cette nécessité repose sur deux principes, orientant la vision du phénomène multiculturel en valeur. Ils se considèrent comme les vecteurs de la paix sociale : l'obligation d'équité et le devoir de réciprocité.

\section{LES MUSULMANS EN EUROPE}

Cette vision peut, par exemple, offrir un cadre théorique des rapports possibles entre les communautés musulmanes vivant en Europe et les sociétés dans lesquelles elles s'inscrivent en tant que «minorité religieuse» ${ }^{1}$.

Or, la présence des musulmans en Europe et leur rapprochement avec l'ordre social läque (neutralité de l'État, séparation des pouvoirs politiques et religieux) soulève de manière 
évidente la dialectique possible du profane et du religieux : entre une société qui, inexorablement, se sécularise et se débarrasse des signes religieux dans l'espace public en les renvoyant à la sphère privée, et un ensemble de musulmans, souvent organisés en corpus, qui se réfère à une spiritualité de chaque instant, sacralise certaines normes et développe une dimension communautaire à leur foi religieuse.

Deux problématiques non similaires, alors, se rencontrent : celle, véhiculée par les pouvoirs publics, de l'intégration nécessaire d'une communauté religieuse minoritaire et marginalisée et du souci de trouver des interlocuteurs restreints pour faciliter le dialogue, et celle véhiculée par les musulmans, soucieux de l'expression libre de leur spiritualité, qui répondent par une forme d'ethnicité symbolique ${ }^{2}$. Majoritairement, leur demande de reconnaissance traduit de puissantes volontés égalitaires. Par ce biais, ils revendiquent un traitement non discriminatoire du temporel de leur culte ${ }^{3}$.

En fonction des considérations historiques et juridiques précises, chaque pays tente de légaliser une formule acceptable (d'une neutralité positive à l'institutionnalisation du religieux), s'orientant dans une logique de différenciation, en partie pour satisfaire aux revendications égalitaires et s'opposer au rejet et à la stigmatisation du groupe que des projections politicoreligieuse et des conditions sociales et professionnelles défavorisées entretiennent.

Mais là où les pouvoirs publics parlent de citoyenneté et d'intégration individuelle, les musulmans insistent sur le respect du lien communautaire. Paradoxal ? L'intégration passe peut-être par le maintien du sentiment subjectif d'une frontière identitaire, même si celle-ci est le fruit d'une construction.

\section{LE CONTEXTE BELGE}

En Belgique, cette hypothèse trouve une plus grande possibilité d'application suite à la spécificité de son contexte historique et juridique : l'État finance les cultes symboliquement reconnus.

Les rapports entre l'islam et le droit commencent ici. L'inégalité de traitement appliqué à l'islam découlait de l'absence d'un organe représentatif, seul obstacle à l'application de la loi de 1974 réglant l'organisation du temporel du culte musulman. L'aspect juridique de la représentation ne fut réglé que tardivement, quatre mois après le scrutin musulman (13 décembre 1998).

La reconnaissance juridique permet au nouvel «Exécutif» de se pencher sur les nombreux dossiers qui l'attendent : le statut des enseignants, la désignation des aumôniers, et plus fondamentalement, l'inventaire des communautés groupées autour des mosquées qui devront graduellement être proposées à la reconnaissance par le Roi.

Car, en effet, l'organe central doit initier un processus graduel de reconnaissance des communautés locales pour que celles-ci, après élection d'un conseil de gestion, puissent prétendre à l'obtention de budgets divers. Ces comités seront autorisés à gérer les intérêts temporels du culte et à représenter localement les fidèles.

Enfin un culte reconnu pourra alors reposer sur des communautés reconnues. Bien plus qu'un «Exécutif» restreint, c'est l'ensemble des comités locaux qui sera idéalement représentatif de l'islam dans le cadre national, rassemblant davantage les spécificités ethniques et les différences doctrinales. La mise en place de l'organe «chef de culte» n'est donc qu'une étape dans la longue route de l'institutionnalisation du religieux. 
L'effet immédiat serait d'abord de démultiplier le nombre d'acteurs musulmans au niveau des différentes bases représentatives et de les désatomiser, de décentraliser la gestion des affaires du culte par une plus grande autonomie de fonctionnement au niveau des provinces (comme c'est le cas pour les orthodoxes) et des communes (comme c'est le cas pour les autres cultes reconnus depuis 1974), et de permettre aux multiples comités locaux d'assurer une orientation moins bureaucratique mais davantage axée sur la religiosité. Les activités proprement religieuses devraient répondre aux difficultés quotidiennes rencontrées par les musulmans.

Alors peut-être ne serait-il plus question d'unifier une idéalité sociologiquement fragmentée, mais au contraire, de la respecter au nom des différences, tout en inscrivant ces dernières dans un contexte commun.

Seules la démocratisation des pratiques de la représentation et la démultiplication des lieux de la centralité religieuse peuvent empêcher que le religieux institutionnalisé ne développe un esprit bureaucratique et technocratique qui le désarticulerait par rapport à sa base.

Mais il reste que le pas à franchir pour passer d'une inconsistante gestion technique et rationnelle des affaires cultuelles à un discours plus véritablement centré sur les questions de fond et les débats de société, trouve obstacle dans l'impossibilité qu'ont les musulmans de développer un clergé idéologique (d'où le nom «Exécutif des musulmans de Belgique», dénué de tout qualificatif) et de laisser s'exprimer sans retenue les gestionnaires (qui «exécutent» un travail administratif) des sas de communication, au nom et place de communautés aussi diversifiées dans leurs sensibilités.

\section{ENTRE RELIGIEUX ET POLITIQUE}

Admettons d'abord qu'aujourd'hui, les musulmans de Belgique, organisés en une myriade de communautés, se sentent honorés par la reconnaissance de leur culte dans l'espace public. Cela n'implique pas forcément qu'ils soutiennent tous l'organe officiel qui les représente symboliquement à l'heure actuelle.

Nous pourrions peut-être imaginer que la composante musulmane, disparate, plus encline au multilinguisme, étrangère majoritairement à l'esprit régionaliste, plus proche par tradition culturelle de l'État uni, et donc plus accoutumée à sa souveraineté temporelle, se considère davantage, de par sa plus grande visibilité, comme partie prenante d'un espace aux composantes culturelles et linguistiques variées.

Inversement, nous pourrions hypothéquer que cette visibilité des musulmans dans le paysage politique et religieux soit facilitée par le contexte particulier d'une société accoutumée à traiter, par le compromis, les différentes composantes communautaires du pays. Après désintoxication des stigmates de l'intégrisme et du mépris religieux, le conformisme aurait vraisemblablement avalisé le changement social stimulé par les acteurs minoritaires.

Que ce soit au sein des diverses institutions politiques fédérées, ou au sein des organes religieux où les dossiers devront être traités de manière spécifiquement adaptée aux réalités linguistiques et politiques des Régions, où la reconnaissance des lieux de culte sera subordonnée à celle des collectivités locales (l'équivalent des fabriques d'églises), elle-même conditionnant le budget qui leur sera alloué, «les musulmans implantés» devront continuer à s'adapter à la réalité complexe de ce pays, faite de pragmatisme et de demi-mesures. Sur le plan institutionnel, a d'ailleurs émergé un islam teinté de «belgitude». 
Leur désir de se sentir membres de la cité à part entière, de jouer un rôle positif dans la société, traduit l'espoir de vivre dignement et tranquillement intégré dans une Belgique plurielle qui reconnaît à chacun le droit d'avoir une opinion et une philosophie de vie.

Donner des carrés musulmans dans les cimetières publics ou reconnaître les deux jours de fête musulmane dans les établissements scolaires, n'est pas une revendication politique mais bien religieuse car l'enjeu n'est pas le pouvoir mais l'aspiration à vivre pleinement sa foi sur un plan individuel.

Or, dans un pays où la politique est omniprésente (administration, magistrature, enseignement...) et où il est difficile de cerner ce qui est politique ou ne l'est pas, il serait illusoire d'imaginer que le rôle des nouveaux acteurs musulmans, plus dépendants administrativement et financièrement de l'État, ne sera uniquement que religieux sans incidences sociales ou politiques. Ils sont, qu'ils le veuillent ou non, dans l'espace politique où se règlent les jeux des pouvoirs, des partis et des groupes d'intérêt.

Si l'imam de telle mosquée exhorte les fidèles à déposer les restes du mouton égorgé dans des containers spéciaux, il aura évidemment un rôle social et politique.

Mais ici la distinction doit être faite entre les actes citoyens et la politique politicienne où le religieux sert de caution à des ambitions de pouvoir.

Sauf dérapages occasionnellement possibles, il est difficile d'imaginer un imam appelant à voter, lors du sermon du vendredi par exemple, pour tel ou tel parti. Les mosquées, certes, expriment fortement les appartenances socio-nationales de leurs membres. Mais elles sont idéalement des endroits neutres («les maisons de Dieu»), réservés à la pratique cultuelle essentiellement, où se célèbre uniquement ce qui unit tous les musulmans dans leur foi, et pas ce qui les divise au niveau de leurs convictions politiques ou autres.

\section{UN BINÓME À RISQUES}

Toutefois, les acteurs musulmans, proches de l'État, par les incidences sociales de leur rôle cultuel ou politique, ne constitueront-ils pas un establishment politico-religieux sophistiqué ? Assisterons-nous à l'émergence d'un nouveau pilier centré sur un communautarisme motivé par une communion de sentiments (ethnicité instrumentale) ou une convergence d'intérêts (clientélisme) ?

D'autre part, la reconnaissance religieuse peut-elle amorcer la volonté d'une représentation politique par des citoyens vivant une communauté de foi ? Au nom du fait religieux, de particularismes quelconques ou de valeurs communes partagées par tous?

Comment peuvent évoluer les relations entre les musulmans et l'État si des éléments religieux portant des ambitions de pouvoir parviennent à démontrer la fragilité de l'intégrité identitaire du groupe, comprimée par des contraintes qu'ils n'acceptent ou qu'ils ne comprennent pas ?

En théorie, l'organe chef de culte représentant plus symboliquement que réellement tous les fidèles, n'a aucune couleur idéologique. Son pouvoir est administratif et si, par ce biais, il se risque à prononcer (ou imposer) des sentences arbitrales sur des points sujets à discussions, elles ne seront vraisemblablement pas respectées par toutes les parties aussi variées que complexes.

Rappelons qu'une des conditions pour poser sa candidature était l'absence de tout mandat politique. Les membres de «l'organe chef de culte», présents d'ailleurs à titre individuel, sont liés par le devoir de réserve de leur opinion politique, comme c'est le cas pour les ministres des 
autres cultes reconnus. De plus, la loi ne permet pas à une entité religieuse d'influencer abusivement dans les actes du pouvoir civil. Les ministres du culte doivent respecter l'autorité publique :

- Seront punis d'un emprisonnement de huit jours et d'une amende de vingt-six francs à cinq cents francs, les ministres d'un culte qui, dans l'exercice de leur ministère, par des discours prononcés en assemblée publique, auront directement attaqué le gouvernement, une loi, un arrêté royal ou tout autre acte de l'autorité publique.» (Article $268 \mathrm{du}$ code pénal).

Le légiférateur s'était donc prémuni contre toute radicalisation des conflits, favorisée éventuellement par le binôme à risques religieux-politique.

Certes, le paysage social a été marqué par de grands mouvements politiques dont l'essence religieuse a orienté la formation de piliers idéologiques. Ceux-ci sont à présent écornés, bien que toujours bien vivants, notamment à travers le tissu associatif.

Quant aux musulmans pourtant, ils devraient plutôt se fondre dans la société belge, la reconnaissance de leur identité collective plaidant pour une participation plus active à la vie de la Cité. Par ce biais, ils pourraient améliorer l'efficacité symbolique du système démocratique.

Si demain, les citoyens partageant une communauté de foi devaient malgré tout être déçus des discriminations à leur égard, tant au niveau de leur participation au sein des différents partis politiques qu'au niveau du traitement accordé au temporel du culte, il n'est pas alors inimaginable de voir se former des regroupements de citoyens d'origine ou de confession commune. Mais alors ce sera le sentiment d'injustice qui servira d'élément moteur à la vision d'un monde divisé en deux, et non une identité religieuse en quête de drapeau. Bien entendu le phénomène de récupération ou de manipulation est possible par certaines élites minoritaires pour qui l'enjeu reste politique ou personnel, et qui peuvent alors exagérer l'importance des contraintes extérieures pour mieux démontrer la perte de l'intégrité identitaire.

Sur le plan religieux, il est évident que le traitement qui sera accordé à l'organe interlocuteur du gouvernement sera conditionné par sa capacité à traiter et faire avancer les dossiers dont il a la charge. Sa légitimité sera sans cesse reposée par la base des communautés et son travail sera jugé par la grande assemblée. Il n'est pas impossible que devant la difficulté à mobiliser les ressources ou en cas de conflits internes, l'Exécutif des musulmans ne puisse pas assurer ses tâches de gestion. Comment réagirait-il alors s'il était sanctionné dans son budget ou qu'il était mis dans l'incapacité de se renouveler librement suite aux contraintes que lui impose l'Etat de droit ? En cas de conflits, religieux et politique musulmans pourraient-ils s'entendre sur une alliance circonstanciée, faisant resurgir les vieux clivages binaires, mêlant les archétypes de la différence et les grands principes universaux, pour faire porter la responsabilité de l'échec sur «le camp adverse»?

Mais dans l'évolution actuelle d'une plus grande visibilisation des musulmans et de l'inclusion des nouveaux acteurs dans la société, il devient incongru de cultiver l'image unilatérale d'une communauté martyrisée et exploitée, en pointant constamment les discriminations dont elle fait l'objet. Le changement social va à l'encontre d'une victimisation qui nierait faussement la possibilité de la classe des «oppresseurs» de ne pouvoir agir sans un seuil d'acceptabilité minimal de ceux qui adoptent une stratégie identitaire de défense. 


\section{LES DIFFÉRENDS ET LEUR GESTION PRAGMATIQUE}

D'une manière très générale, il faut malgré tout admettre que la mixité des différences, par leur hybridation culturelle dynamique, produit immanquablement, tôt ou tard, des incompréhensions et des différends.

$\mathrm{Au}$ nom d'une nécessité pragmatique, des techniques visent à rétablir la communication, recréer la solidarité et restaurer la paix sociale : la conciliation, l'arbitrage et la médiation. S'instruisant du sens singulier de chaque situation, animée d'une forte sollicitude pour autrui, emplie de sagesse, la médiation tente de trouver le point d'équilibre entre civilité et altérité, appelant au respect de cette dernière pour que l'osmose communicative soit plus performante.

Dans le cadre des relations entre l'État et les musulmans, l'esprit de médiation est rendu nécessaire en raison du différend ressortant du «décalage sémantique» entre la demande d'intégration des uns, animée également d'intérêts sécuritaires au nom de l'État de droit, et celle du traitement non discriminatoire des autres, animée de liberté d'expression religieuse au nom du droit pour tous.

Pour ne pas être accusée de servir les intérêts dominants et de relayer une ingérence menaçant l'intégrité identitaire, la médiation doit être anticipée et dynamisée civiquement à partir du milieu musulman. L'important n'est pas de s'identifier aux revendications des uns et des autres, mais de réfléchir sur des propositions actives et constructives dans un climat de concertation.

Les médiateurs n'ont donc pas de vrai pouvoir. Ils doivent prétendre à un maximum d'indépendance et de neutralité...et le prouver par une promesse de retrait, la médiation accomplie. La confiance est adressée à ce prix et les marques de désintéressement conditionnent le pouvoir d'autorité nécessaire pour que les engagements pris de part et d'autre soient respectés.

\section{UNE MÉDIATION «MUSULMANE»?}

La médiation aidée par les conciliateurs des deux camps dépasse alors le cadre normatif et idéologique. Elle est néanmoins «musulmane», car la forme qu'elle prend ne peut échapper à la sélectivité et à la priorité des valeurs choisies par référence.

En plaçant, par exemple, la justice comme une valeur absolue, au-delà de tous les clivages ethniques, raciaux et communautaires, le pragmatisme et les actions rationnelles sont orientées en valeur. L'impartialité de la justice est rappelée à partir des sources traditionnelles (Coran, tradition prophétique), et symbolisée par «l'alliance des braves» (hilf al-fudul $)^{4}$.

L'espace neutre de rencontre est alors redéfini à partir d'une socialité, expression moderne de la futuwwah ${ }^{5}$, mêlant sens du devoir, sollicitude pour autrui et aspiration à une justice impartiale, avec laquelle les acteurs musulmans, de l'intérieur, donnent un sens à leur action sociale. Il est marqué également par un avalement sémantique imposé, de l'extérieur, par les modifications du champ culturel : des concepts extérieurs à la $d_{i n}{ }^{6}$ sont utilisés et intégrés (démocratie, responsabilité citoyenne, contrat social, éthique de l'action...), lorsqu'ils peuvent s'appuyer, dans une translation variable de sens, à partir du message religieux ('adl et quist, masuliyya, 'ahd, 'amal...).

L'alchimie opérée par les médiateurs musulmans ne se réduit pas à rechercher les universaux culturels ou à appeler au respect des différences. Loin d'essentialiser ces dernières, il les combine, exalte leurs qualités, les valorise, dégage les énergies latentes, de manière à les 
amener, par autotransformation et recomposition, à un stade plus avancé dans la résolution des différends.

Les situations conflictuelles sont alors davantage désintoxiquées des paradigmes exaltant les différences ségrégatives dans les deux camps. Une présentation plus adaptée est formulée, en usant davantage d'une raison humaine fondatrice de la communicabilité du discours que d'une ethnicité instrumentale, en employant un langage qui interpelle les uns et les autres, en sensibilisant aux vertus des discussions, en rationalisant les dissonances cognitives et les contraintes, en recontextualisant ce qui alimente les fictions collectives.

Les médiateurs tentent le mariage des différences destinées à «l'entre-connaissance» ${ }^{7}$. Ils visent à dépasser le modèle binaire «dominant de droit/dominé de fait», entretenu par ceux, d'une part, qui baignent dans une sollipcisme culturel et s'indignent de manière sélective, et ceux, d'autre part, qui se victimisent et s'emprisonnent dans une culture d'aliénation.

Ils insufflent, au sein des espaces de rencontre, un état d'esprit d'ouverture dans lequel l'obligation d'équité et le devoir de réciprocité s'éveillent davantage suite à l'acceptation et au partage des responsabilités dans la genèse et la résolution potentielle des différends, sans coupables, ni procès, ni bourreaux, ni victimes, sans imposer une représentation unificatrice du temps et de l'espace, ni déprécier les cultures par une donnée fixe et permanente de l'identité culturelle qui enferme les uns et les autres dans leurs différences, sans aussi procéder de l'obsession à la pureté, ni de la prétention vaniteuse à l'ultralucidité et à l'authenticité.

\section{CONCLUSION}

L'esprit de médiation, principalement animé par les nouveaux inclus, quelle que soit la forme de son cadre, participe, dans une société qui connaît une dynamique plurielle de construction dans le sens d'un rapprochement des uns et des autres (l'islam et l'Etat, par exemple), à catalyser l'inter-connaissance en plus grande réciprocité au nom d'une justice impartiale. Elle aide aussi à gérer les réactions de défense suscitées par la crainte de la fonte identitaire quand la diversité aménagée vit une situation fragile face aux contraintes que la vie à plusieurs implique, surtout quand celle-ci sont perçues comme uniformisantes.

\section{NOTES}

En nombre de fidèles.

2 Les musulmans deviennent visibles, par leur action, dans l'espace public.

3 Aspects liés à la gestion administrative et financière du culte : enseignement et formation, aumônerie, gestion des lieux de culte et exonération fiscale, parcelles d'inhumation, accès aux médias, jours de fête...

4 Serment prononcé à La Mecque, en compagnie du Prophète (avant qu'il ne reçoive sa mission), pour venir en aide aux nécessiteux, indépendamment du clan auxquels ils appartiennent.

5 Par extension du sens littéral, «noblesse de cœur», équivalent à la notion de» chevalerie».

6 Terme utilisé pour définir l'ensemble des représentations et des pratiques liées à une religion.

7 «Oh hommes! Nous vous avons créés d'un mâle et d'une femelle, et nous avons fait de vous des nations et des tribus, pour que vous vous entre-connaissiez...» (Coran, 49/13). 